\title{
Low cost TVAC Chamber for aerospace tests
}

\author{
Juan Manuel López Torralba \\ GranaSAT Aerospace Group \\ University of Granada \\ Granada, Spain \\ jmlopezt@correo.ugr.es
}

\author{
Andrés Roldán Aranda \\ Electronics Department \\ University of Granada \\ Granada, Spain \\ amroldan@ugr.es
}

\begin{abstract}
All the spacecraft subsystems must be exhaustively tested before launch in order to obtain space-worthy solutions. The aim of this paper is to perform several qualification tests for increasing the Technology Readiness Level (TRL) of a Commercial Off-The-Shelf (COTS) solar cell protection diode. For accomplishing the tests, we undertake the design and manufacturing of a Thermal Vacuum (TVAC) Chamber able to reach extremely low temperatures and to deal with High Vacuum (HV) conditions. This testing facility system will be able to recreate space conditions and to perform aerospace tests.
\end{abstract}

Keywords - TVAC Chamber; High Vacuum; Low cost; Spaceworthy components, Technology Readiness Level, Solar cell protection diodes.

\section{INTRODUCTION}

Before flying a satellite, thoroughly environmental tests must be performed to guarantee mission success and survivability of the whole system. These tests are carried out in dedicated equipment denominated as Thermal Vacuum (TVAC) Chambers. A TVAC is used to reproduce as closely as possible the space conditions which a satellite will be exposed to. The closest equipment which can be found in most electronic laboratories is the environmental chamber. However, the common climatic test chambers are not able to perform test in vacuum and below $20^{\circ} \mathrm{C}$.

The TRL of a spacecraft component is classified on a scale from 1 to 9 and defines its technical maturity. In this research our target is to increase the TRL level of a COTS diode not to flight-proven level (TRL9) but to functional verification level (TRL4).

The TVAC design is proposed as a low-cost alternative to commercial solutions found in the market. In order to simulate the system, it was needed in-depth knowledge of computer-aided design (CAD), computer-aided manufacturing (CAM) and thermal analysis. The use of this TVAC chamber in the Aerospace Electronic course of the Telecommunication Master for testing a COTS solar cell protection diode has introduced students in vacuum technology and improved their skills in aerospace measuring procedures.

\section{THERMAL VACUUM CHAMBER}

\section{A. Structure}

The main purpose of this research consists in the design of an economical TVAC chamber. There are a wide range of structural shapes for TVAC chambers ranging from simple boxes and cylinders to spheres and geodesic balls [1]. The shape which presents the best structural rigidity and minimize material is a sphere [2]. However, a box shaped TVAC chamber has been designed considering the manufacturability, internal dimensions and the final expenses.

The TVAC chamber has been designed with $260.4 \mathrm{~kg}$ in weight and 372.71 in capacity, able to reach HV conditions and extremely low temperatures. AISI 304 stainless steel was the material preferred for the parts that are exposed to vacuum such as the chamber walls, internal shroud, doors and ports considering its outgassing rates and its high strength [4]. AISI 304 Stainless Steel (SS) $10 \mathrm{~mm}$ thickness has been used for the manufacturing of the chamber walls [3].

The chamber has two configuration possibilities for the vacuum chamber door. The first one consists of a $40 \mathrm{~mm}$ in thickness methacrylate door which allows observing the aerospace test without pressure leakages. Additionally, there is a $10 \mathrm{~mm}$ thickness AISI 304 stainless steel door with a $90 \mathrm{~mm}$ diameter viewport in its center. To attach the viewport into the door a $10 \mathrm{~mm}$ in thickness washer shape supplement has been added in order to gain thickness in the mounting. The claw clamp bolts go into tapped holes on the supplement. To avoid leakages in the viewport, a total of eight single claw clamp has been added. The use of less clamps results in a likelihood of leakage.

In Fig. 1 and isometric views of the designed TVAC Chamber with methacrylate and 304 stainless steel vacuum gate configuration are shown, respectively.

The internal structure of the chamber contains a shroud which outer part is covered with MLI film to reduce cooling time[4]. Between the MLI film and the outer faces of the shroud a piping system has been added. The shroud consist of an AISI 304 stainless steel $2 \mathrm{~mm}$ in thickness squared structured with dimensions of $650 \times 650 \times 525 \mathrm{~mm}$ and a rail guide. The rail guide is used for accessibility purposes. Additionally, several crossbars have been added in the outer faces of the chamber to increase the structural rigidity [2] and to benefit from improved maneuverability of the TVAC chamber. The support base is responsible for preventing vibrations from the vacuum and thermal systems [1]

The TVAC chamber includes multiple feedthrough ports for instrumentation purposes and for the normal chamber operation avoiding any pressure leakage or thermal change on the running tests. All the ports are sealed with NW Wing Nut 
Clamps and O-rings to reduce thse probability of failure. There are a total of seventeen feedthrough ports including three NW-16 Type-K Thermocouple and three NW-25 8 pin Electrical Instrumentation Feedthroughs. These feedthrough are able to transmit $1 \mathrm{kV}$ signal voltages and currents up to 7 A. In addition, a total of two KF-16 and seven KF-25 welding sockets have been added to meet future needs. Finally, two dual liquid nitrogen $\left(\mathrm{LN}_{2}\right) \mathrm{KF}-50$ feedthroughs have been included for the injection and extraction of $\mathrm{LN}_{2}$ to the Chamber. The ports which are not in operation are ended with blank flanges.

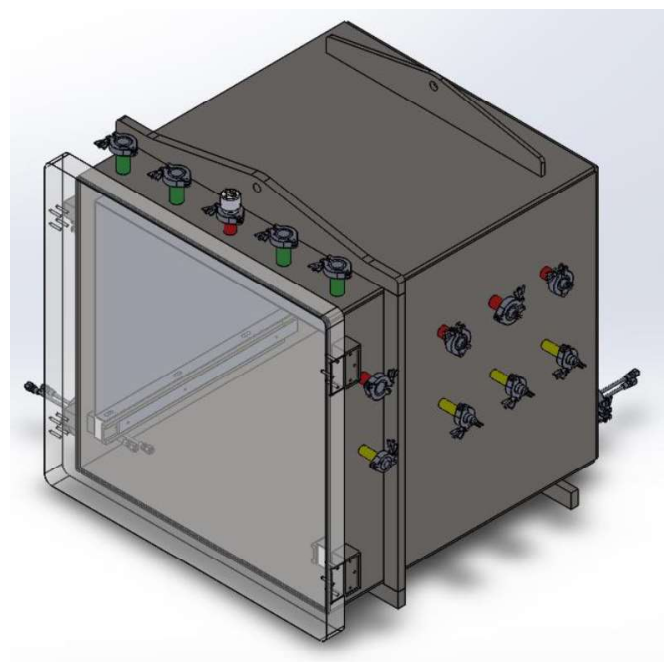

Fig. 1. TVAC Chamber with the methacrylathe door.

In the Table 1 a list of features of the designed TVAC chambers are shown.

TABLE 1. LIST OF FEATURES OF THE TVAC CHAMBER
\begin{tabular}{|l|c|}
\hline Shape & Box \\
\hline Inner Dimensions & $700 \times 745 \times 700 \mathrm{~mm}$ \\
\hline External Dimensions & $1065 \times 1024 \times 857 \mathrm{~mm}$ \\
\hline Internal Volume & 372.751 \\
\hline Shroud Dimmensions & $650 \times 650 \times 525 \mathrm{~mm}$ \\
\hline Structural Material & AISI $304 \mathrm{SS}$ \\
\hline Flanges Material & Stainless Steel \\
\hline Flange Type & ISO KF-16/25/50/63 \\
\hline Gate Configuration & Methacrylate $\& 304 \mathrm{SS}$ \\
\hline Viewport Type & Kodial Optic \\
\hline Viewport Diameter & $90 \mathrm{~mm}$ \\
\hline Weight & $260.39 \mathrm{~kg}$ \\
\hline
\end{tabular}

\section{B. Vaccum System}

The objective of the vacuum system is to decrease the pressure within the chamber to a desired level in a brief period of time on a clean atmosphere. The designed TVAC Chamber is able to deal up to $10^{-5}$ Torr. This vacuum condition must be maintained during the whole test. The pressured within the chamber will be measured with a 925 MicroPirani Vacuum Transducer controlled via RS-232.

The vacuum system consist of two interconnected pumping units [1][3][4]. First, a Telstar rotary vane pump is used to reduce the pressure inside the TVAC chamber from ambient to
$10^{-3}$ Torr [4]. For releasing the pressure from $10^{-3}$ Torr down to $10^{-5}$ Torr a Turbo-molecular pump is used [3]. The Turbo-molecular pump is only needed in cases in which the $\mathrm{HV}$ conditions are a requirement for the test. It is connected to the Chamber using a dedicated ISO-63 feedthrough port located on the backside. The cooling of the Turbo-molecular pump is carried out by the Isotemp 6200 R35 Recirculating Chiller.

The rotary vane pump is an oil-sealed positive rotary displacement pump. The pumping system consists of vanes mounted to an eccentrically installed rotor inside of a circular housing. The Turbo-molecular pump consists of rotor and stator blades. The transfer of movement stimulus from the swiftly rotating blades to the gas molecules being pumped is the base of the pumping effect.

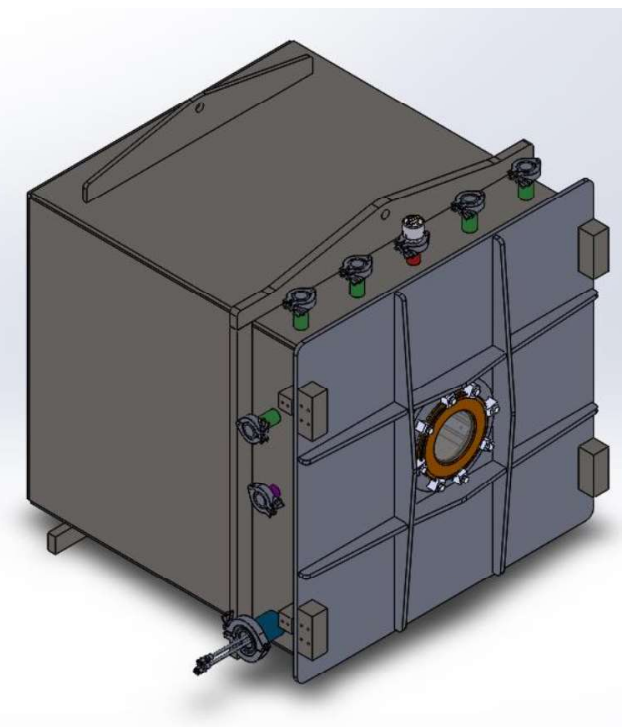

Fig. 2. TVAC Chamber with the 304 stainless steel door

The use of two pumping units is the consequence of the performance limitations of the pumping units available in the market.

The block diagram of the Vacuum System is shown inFig. 3. Vacuum System block diagram.Fig. 3.

\section{Thermal System}

The objective of the thermal system is to recreate the extremely low temperatures in space. The system must have total control of the temperatures inside the chamber during the thermal tests. The temperatures of different elements of a satellite are collected using three NW-16 Type K thermocouples. We expect to obtain precious data for further understanding of the thermal responses of a satellite expose to the space environment.

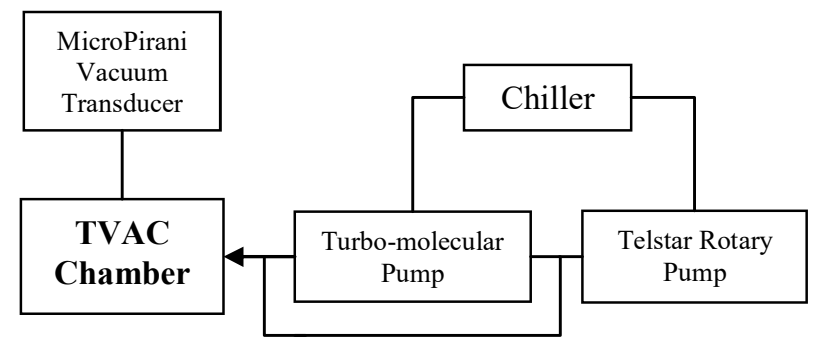

Fig. 3. Vacuum System block diagram. 
The TVAC chamber implements a thermal control mode which consists of Cooling and Heating stages [4]. For the first one it can be used $\mathrm{LN}_{2}$ injection or the Isotemp $6200 \mathrm{R} 35$ Recirculating Chiller, reducing the inner temperature of the chamber down to $-180^{\circ} \mathrm{C}$ and $-35^{\circ} \mathrm{C}$, respectively. The $\mathrm{LN}_{2}$ is injected into the chamber using the Dual liquid Nitrogen Feedthrough located on the back of the TVAC chamber. The $\mathrm{LN}_{2}$ is then discharged as gas through another Dual liquid Nitrogen Feedthrough located on the lateral of the chamber. These feedthroughs are built with dual coaxial tubes which efficiently reduces condensation on its external side ensuring the integrity of the seal. As for the heating stage, the same Recirculating Chiller can used to swiftly heat the shroud up to $200^{\circ} \mathrm{C}$. In both stages a copper piping system covered by MLI film is used for the circulation of the fluid [4]. The block diagram of the Thermal System is shown in Fig. 4.

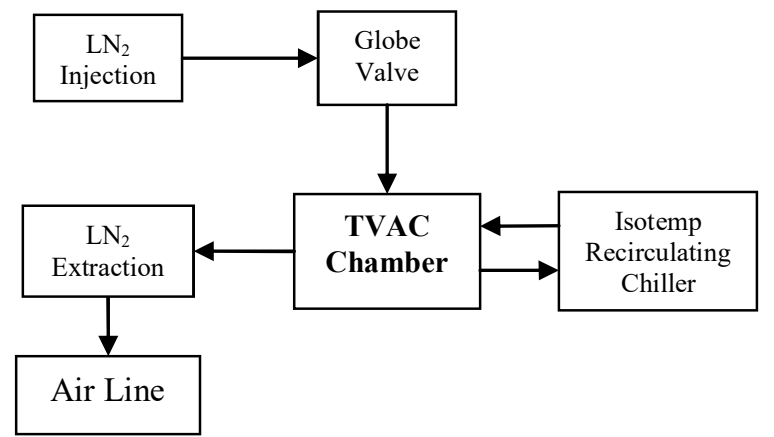

Fig. 4. Thermal System block diagram

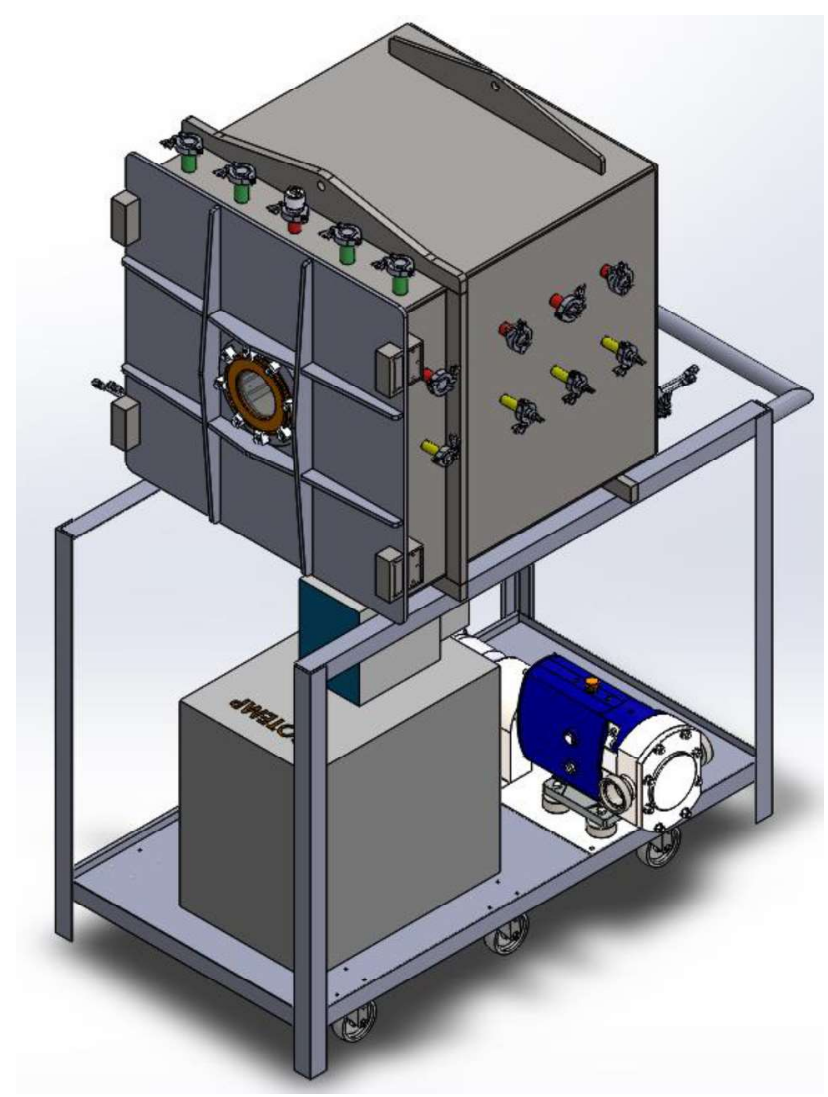

Fig. 5. Thermal Vacuum Chamber front view.

All the temperature parameters involved in the thermal tests are controlled externally using a computer with Matrix Laboratory (MATLAB) GUI measurement application designed by the students.

\section{Instrumentation Data Acquisition and Control System}

The Control System implements the interfaces and tools for controlling and supervising all the electronic and mechanical devices and systems which constitute the designed TVAC chamber [1]. The Instrumentation Data Acquisition is a critical stage for successfully perform aerospace tests. The more data collected the better for the comprehension of the satellite behavior in the space environment. Controlling each stage of the vacuum and thermal cycles is possible with a computer and MATLAB. The designed TVAC Chamber has a total of seventeen feedthrough ports for instrumentation purposes. This data provides the engineers an adequate amount of information for supervising the processes or even intervene in the ongoing operation if needed. If desired, further analysis of the under test satellite can be done in order to minimize risk in its development. The 12 multiplexed channel Siglent SDM3065X $61 / 2$ Digits Dual-Display Digital Multimeter is used through USB to measure temperatures in different internal points, currents and voltages selected by the user.

As said before, three feedthrough ports with eight pins are used for sink or drain current or voltage signals up to $1 \mathrm{kV}$ and $7 \mathrm{~A}$. For the thermal analysis another three Type K Thermocouple feedthrough ports are used. The unused ports are able for expansion purposes. In Table 2 a list of the functionalities of the feedthrough ports can be found.

\begin{tabular}{|c|c|c|}
\hline Feedthrough port & Quantity & Functionality \\
\hline \multirow{2}{*}{$\begin{array}{l}\text { KF50 Dual Liquid } \\
\text { Nitrogen }\end{array}$} & \multirow{2}{*}{2} & $1 \times \mathrm{LN}_{2}$ Injection \\
\hline & & $1 \mathrm{x} \mathrm{\textrm {LN } _ { 2 } \text { Extraction }}$ \\
\hline \multirow{3}{*}{ KF25 Half Nipple } & \multirow{3}{*}{5} & $\begin{array}{l}\text { 1x MicroPirani Vacuum } \\
\text { Transducer }\end{array}$ \\
\hline & & $\begin{array}{l}3 x \text { Electrical } \\
\text { Intrumentation }\end{array}$ \\
\hline & & 1x Future Needs \\
\hline $\begin{array}{l}\text { KF25 Long } \\
\text { Nipple }\end{array}$ & 1 & Recirculating Chiller \\
\hline \multirow{3}{*}{$\begin{array}{l}\text { KF16 Metric } \\
\text { Long Nipple }\end{array}$} & \multirow{3}{*}{5} & 3x Type K Thermocouples \\
\hline & & 1x Telstar Rotary pump \\
\hline & & 1x Recirculating Chiller \\
\hline $\begin{array}{l}\text { KF25 Metric } \\
\text { Long Nipple }\end{array}$ & 5 & Future Needs \\
\hline ISO63 Nipple & 1 & Turbo-molecular Pump \\
\hline
\end{tabular}

\section{SOLAR CELL PROTECTION DIODES TESTS}

\section{A. Burn In (BI)}

The BI qualification test purpose is to significantly reduce operational failure rate by screening out infant mortality failures within the qualification batch [5].

The process consists of two phases consisting of $200 \mathrm{~h}$ reverse biases with $\mathrm{V}_{\mathrm{REV}}=-1.5 \mathrm{~V}_{\mathrm{OC}}$ and $16 \mathrm{~h}$ forward biased with $\mathrm{I}_{\mathrm{FW}}=1.1 \mathrm{I}_{\mathrm{SC}}$, respectively, under ambient pressure conditions. In both phases the diode must be under load with a temperature of $\mathrm{T}_{\mathrm{J}}=\mathrm{TBS}^{\circ} \mathrm{C}[5]$.

\section{B. Temperature Behaviour (TB)}

The TB qualification test purpose is to determine the electrical parameters of the solar cell protection diodes as a function of the temperature in order to design the solar generator [5]. 
The process consists of performing the Diode Characterization (DC) test at three temperatures as a minimum. Those temperatures are $\mathrm{T}_{\mathrm{J}}=25^{\circ} \mathrm{C}, 80^{\circ} \mathrm{C}$ and $150^{\circ} \mathrm{C}$ for common test. For mission specific qualification, those temperatures changes according to the diodes highest, operational and lowest predicted temperature [5].

\section{Diode Characterization (DC)}

The DC qualification test purpose is to analyze the performance degradation of the diode after an environmental test [5].

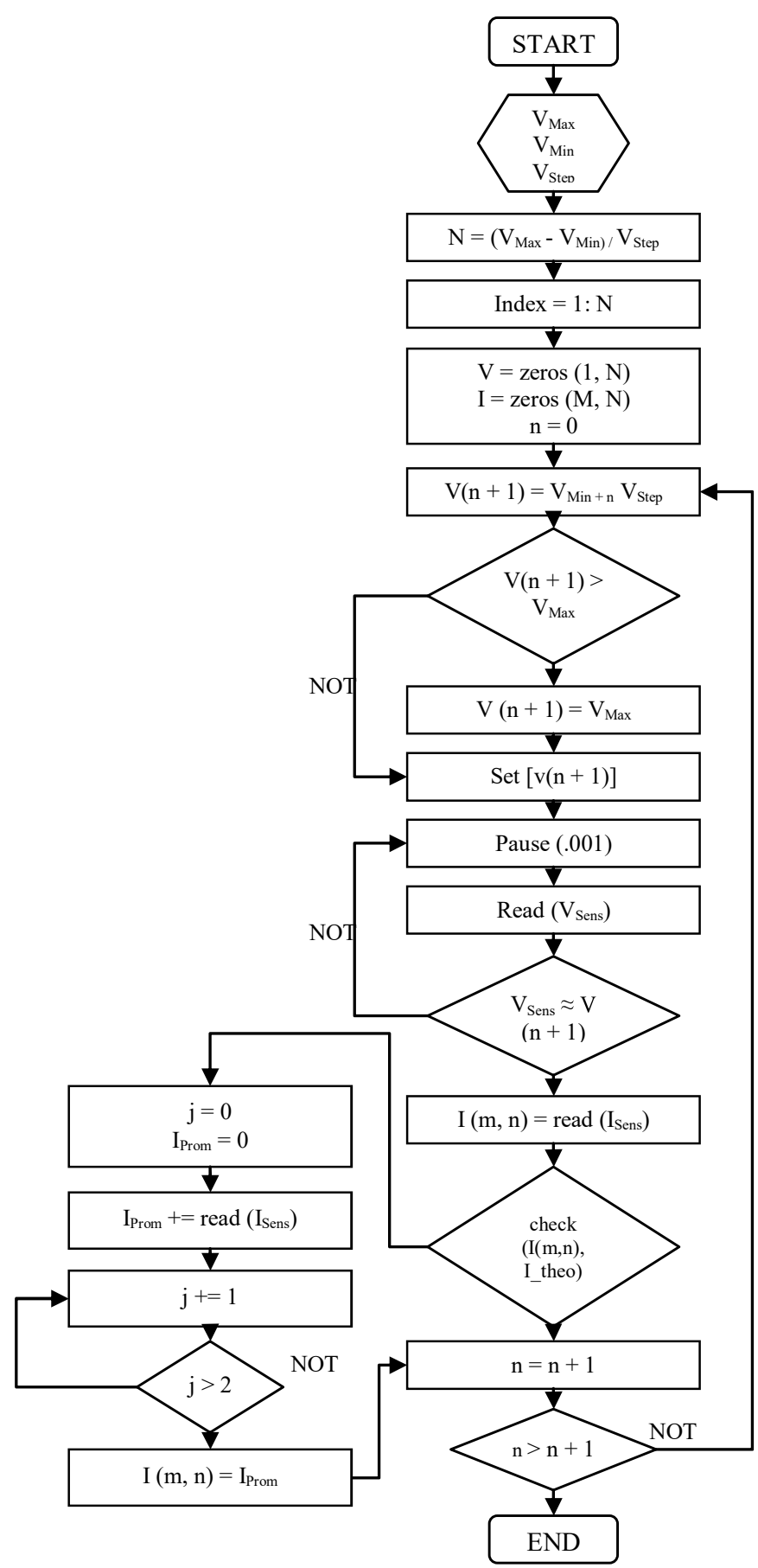

Fig. 6. Diode Characterization Qualification Test
The procedure is based on obtaining the forward and reverse $\mathrm{I} / \mathrm{V}$ characteristic of the protection diode. For performing the test, the forward current must be 1.1 times the expected current at the maximum mission temperature. For the reverse stage, the reverse voltage must be $\mathrm{n}$ times the expected solar cell $\mathrm{V}_{\mathrm{OC}}$ voltage at the minimum mission temperature, where $\mathrm{n}$ ranges from 1.33 to 1.5 .

The qualification test must be recorded at different temperatures, including the maximum and minimum performing temperatures for different times [5]. In the Fig. 6 a block diagram of the DC test is shown.

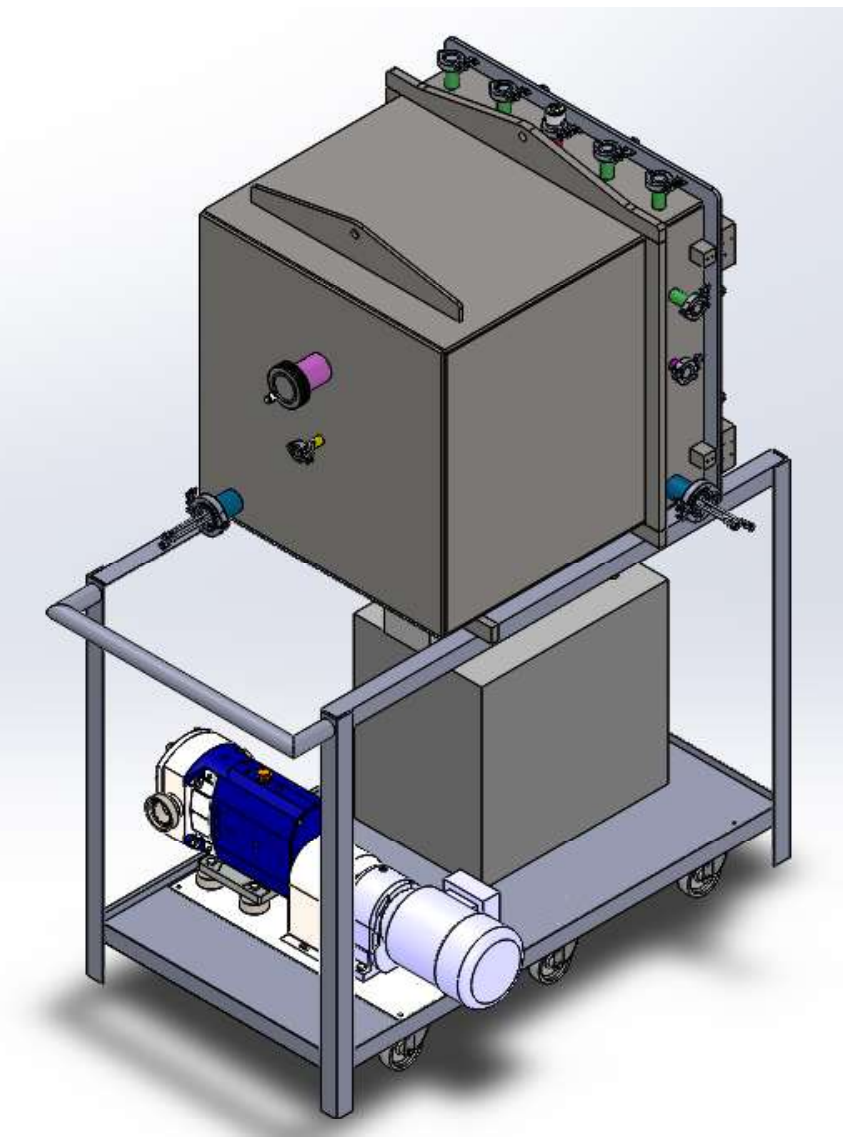

Fig. 7. Thermal Vacuum Chamber rear view.

Stainless steel has been used for the manufacturing of the Chamber, the internal shroud and the feedthrough ports. The designed system has a total capacity of 372.751 , figure 7 . The capacity of the inner chamber is 221.81 1. A Vacuum System has been designed with the capability of reaching Medium and High Vacuum. High Vacuum is attained in two steps configuration using Turbo-molecular and rotary pump in series. If working in Medium Vacuum (MV) conditions the rotary pump is used directly. The designed Thermal Vacuum allows reducing the internal temperature to $-160^{\circ} \mathrm{C}$ using $\mathrm{LN}_{2}$. A shroud covered by MLI film is used in order to decrease the time of cooling. 


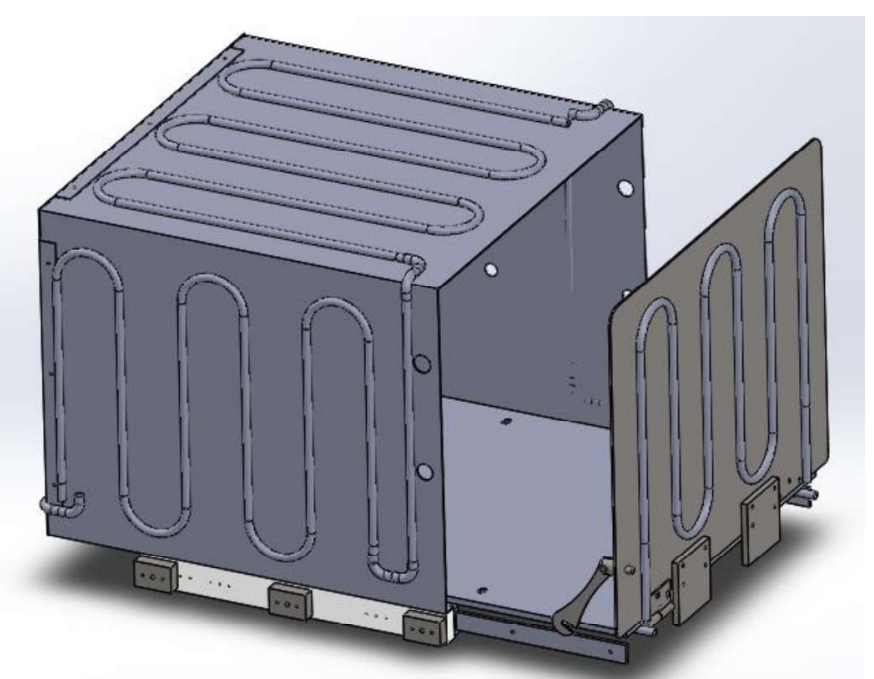

Fig. 7. Shroud and dual thermal liquid circuit.

Priceless instrumentation data will be obtained through the aerospace test performed onto the TVAC Chamber. This information will be greatly appreciated for the engineering teams involved in the developed and testing of satellites under analysis. We also expect to reduce expenses in development stages. The use of the manufactured TVAC Chamber for aerospace testing will be crucial for avoiding critical mistakes.

Finally, a preliminary 24 hours-length vacuum test has been performed in the TVAC Chamber reaching MV (1-0.01 mbar) conditions. The rotary pump has been used directly for this test. Our MATLAB control panel has been used for the signals acquisition. In Fig. 8 the results of the test can be observed.

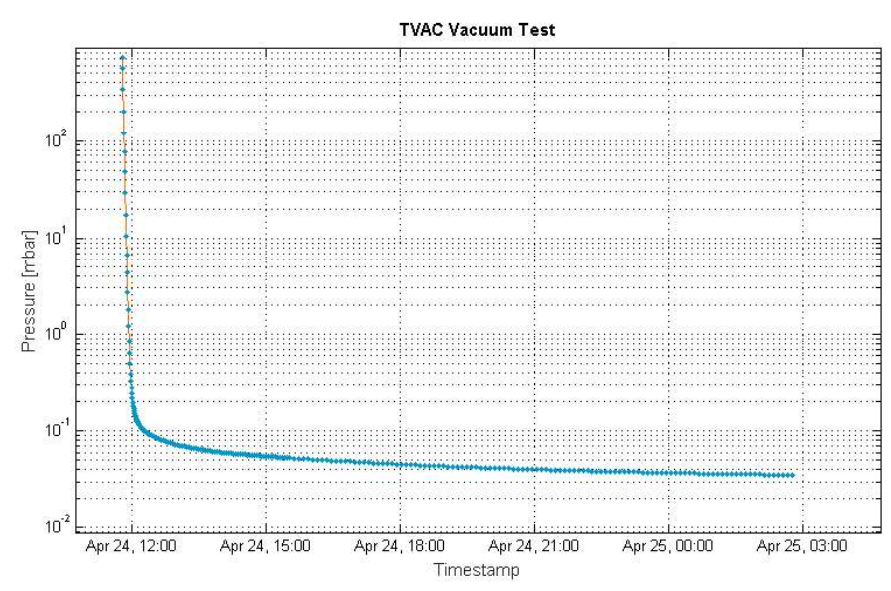

Fig. 8. TVAC MV Test not including Turbo-molecular pump.
In the above figure we can observe the TVAC reaching MV conditions after 1 hour.

\section{CONCLUSIONS}

This research describes the design and construction of an economical and high performance Thermal Vacuum Chamber able to operate aerospace test. The idea of designing a TVAC Chamber arise as a reaction to the existing TVAC chambers on the market, where prices are not affordable for modest research groups. However, for some years now, the design of modest vacuum chambers has been breaking barriers in terms of price and performance [1][3][4]. Our research is based on these investigations and aims to contribute in the design of economical and high performance vacuum chambers. Besides, the use of the designed TVAC Chamber in other research fields such as biomedicine or biology is promising.

The total cost of the design and manufacturing has been less than $3000 €$ which is an affordable cost compared with the available market solutions. We hope this research will act as source of inspiration for researchers who wish to manufacturing similar equipment to their laboratories.

The Graphical User Interface application based on MATLAB has been developed by students in order to perform all the measurements of physical magnitudes (temperature, current, voltage, etc.). The usage of the TVAC chamber in the lab. activities is a valuated task by the students.

\section{ACKNOWLEDGMENT}

The experiments and the TVAC Chamber fabrication were supported by GranaSAT Aerospace in collaboration with the Telecommunication Master Student office. The authors also gratefully acknowledge the important contribution of lab technicians of the company Mecanizados Granada S.L.

This research received financial support from the national government of Spain, Project DEEPSAT RTC-2016-4644-3.

\section{REFERENCES}

[1] R. Stevenson et al., "Development of a Thermal-Vacuum Chamber for testing in Small Satellites," pp. 2017-228.

[2] K. Harrison, "Engineering a Better Vacuum Chamber," pp. 1-4.

[3] S. Jayaram and E. Gonzalez, "Design and construction of a low-cost economical thermal vacuum chamber for spacecraft environmental testing," J. Eng. Des. Technol., vol. 9, no. 1, pp. 47-62, Mar. 2011.

[4] J. Jeon et al., "Construction of a thermal vacuum chamber for environment test of triple CubeSat mission TRIO-CINEMA," J. Astron. Sp. Sci., vol. 30, no. 4, pp. 335-344, 2013.

[5] "Space engineering Photovoltaic assemblies and components ECSS Secretariat ESA-ESTEC Requirements \&amp; Standards Division Noordwijk, The Netherlands," 2012. 